\title{
BOUNDS FOR EFFECTIVE MATERIAL PROPERTIES OF STATISTICALLY NON- HOMOGENEOUS SOLID
}

Muneo HORI*

\begin{abstract}
The prediction of the effective properties of heterogeneous materials is of primary importance in design or analysis. For statistically nonhomogeneous materials, the prediction is laborious since the effective properties vary depending on various conditions. This paper presents a method of estimating bounds for all possible effective properties for given limited information. Considering permeable flow through rock mass as an example, the bounds are rigorously derived and are compared with ranges obtained from numerical simulation of heterogeneous porous media. The results support the validity of the proposed method.

Key Words : effective material properties, bounds for effective properties, micromechanics, statistical homogeneity, permeability of rock mass
\end{abstract}

\section{INTRODUCTION}

In general, a natural geomaterial such as rock or rock mass is highly heterogeneous. In design or analysis, however, they are modeled as homogeneous with effective material properties that manipulate their overall behaviors or responses. This is called homogenization; see Nemat-Nasser and $\operatorname{Hori}^{1,2)}$, for an extensive survey of literatures related to the homogenization. The precise prediction of the effective material properties is essential in making the design or analysis more rational. For the homogenization of natural heterogeneous materials, one may encounter the following two major problems: 1) the geological structure of the geomaterials is not uniform, varying in an almost unpredictable manner; and 2) the information on the heterogeneities is often limited due to the difficulty of extensive field measurements; see, for example, Bear and Buchlin ${ }^{3,4)}$, $\mathrm{Oda}^{5(7)}$ and Long and Witherspoon ${ }^{8}$ in rock hydraulics. In consequence, it is laborious to estimate the effective properties which change place to place in a relatively large domain.

For man-made heterogeneous materials, such as composites or metal alloys, the situation is less complicated ; they are processed to have similar microstructure everywhere, and necessary information is obtained from laboratory experiments. Hence, while the prediction of the effective properties have been studied and a huge amount of knowledge have been accumulated for such artificial heterogeneous materials ${ }^{9)}$, the direct application of the established methodology is not feasible, unless some special assumptions are

\footnotetext{
* Department of Civil Engineering, Tokyo University
}

made; see Bear and Buchlin ${ }^{3)}$ for the detailed explanation of assumptions necessary in analysis of flow through natural porous media.

The prediction of a single value for the effective material property is difficult, when the data on the heterogeneities are limited. For the practical purpose, it is desirable to predict the range of all the possible effective properties as well as a single value that is the most likely to occur. If the extreme value determined from the range is used, design or analysis provides a limit state of the best or worst case of behaviors of the heterogeneous material. Although statistical analysis is required for the prediction of the range, one cannot ignore the mechanism that determines the overall behavior of heterogeneous materials, i.e., the effective properties are determined through the relation between the average field quantities, and hence mere volume averages of local material properties do not provide an accurate estimate of the effective properties when the difference in the local properties is relatively large.

In various fields of mechanical, electromagnetical, or optical engineering, the need for more sophisticated treatment of heterogeneous materials is increasing with the aim of processing advanced materials with superior properties and conducting efficient numerical analysis of structures made of such materials. To satisfy this demand, existing theories ${ }^{9)-12)}$ are unified and more general theories are being constructed ${ }^{1,2)}$. Based on the recent achievement, this paper proposes the method of predicting bounds for effective material properties of a general heterogeneous material which has microconstituents distributed in an arbitrary manner and information for them limited. The key issue in making the prediction more accurate is 
rigorous treatment of local field variables.

To make access to manipulation easier, this paper considers a problem of permeable flow through rock mass as a direct example, although the proposed method is general and can be applied to other problems. It should be noted that the estimate of the effective permeability of rock mass is one of the current concerns in researches of determining a deposit site for nuclide wastes. The structure of this paper is constructed as follows: first, several remarks on the effective permeability of highly heterogeneous rock mass are stated in Section 2. Then, in Section 3, exact inequalities which constrain the effective permeability are rigorously derived from physical field equations, and Section 4 computes the bounds using the inequality and obtains the closed-form expression for them. In order to demonstrate the validity of the proposed method, Section 5 presents the results of numerical simulation for flow through randomly generated porous media. It is shown that all the computed effective permeabilities lie between the estimated bounds.

\section{HOMOGENIZATION OF ROCK MASS}

It is intuitively acceptable that overall behaviors of a sufficiently large sample ${ }^{\text {footnote1) }}$ determine the effective property of a heterogeneous material. Hence, a member or a structure made of this material can be modeled as one with the identical geometry but consisting of a fictitious homogeneous material with the effective properties if the microstructure pattern is the same throughout the member or the structure. This condition is statistical homogeneity. There are various strict definitions of the statistical homogeneity, by making more clear phenomenological observation $^{14)}$, considering correlation between the locations of distinct heterogeneities ${ }^{11), 13)}$, combining the stationarity and ergodicity of field variables ${ }^{15)}$, or applying asymptotic expansion as the microconstituent size vanishes ${ }^{16) \sim 18)}$.

For natural geomaterials, however, the statistical homogeneity does not hold since the geological structure can vary in a larger region. The distribution of geological constituents is statistically nonhomogeneous; see Fig.1. Indeed, for natural porous materials, Bear and Buchline ${ }^{3)}$ require a subregion for homogenization to be sufficiently smaller than the length scale of the geological structure changes. If a material is statistically nonhomogeneous, even larger samples do not

\footnotetext{
footnote1) Such sample is called a representaive volume element, though rock hydraulics ${ }^{3), 4)}$ uses a term representative elementary volume.
}

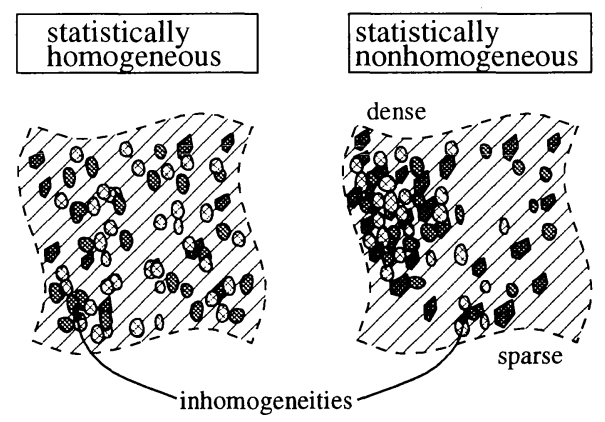

microstructure is more or less similar every place microstructure changes place to place
Fig.1 Statistical homogeneity and statistical nonhomogeneity

exhibit common overall behaviors. Furthermore, the responses of one sample change depending on loading conditions ${ }^{2}$. Hence, effective properties lie in a relatively large range.

In particular, flow through rock mass passes joints or cracks which are distributed and connected in a statically nonhomogeneous manner. While $\mathrm{Oda}^{5,6)}$ has proposed a rational method of estimating the effective permeability in terms of crack tensor, such homogenization is not applied when, say, nuclide migration through groundwater in a large region and in a long time span is considered to assess the safety of the surrounding environment. At the current stage, fracture network analysis or a Monte-Carlo simulation for flow through connected joints is usually applied; see, for example, Long and Witherspoon ${ }^{8)}$. In fracture net work analysis, various routes of connected joints or cracks, which are randomly generated to meet geological nature of the rock mass, must be examined.

There is no doubt on the advantage of homogenization (if possible) when one makes design or analysis of underground structures in rock mass using large-scale numerical computation. The difficulty of homogenization is the statistical nonhomogeneity of rock mass, which implies the uncertainty of the effective permeability. As an alternative, one may consider the best or worst case for the permeable flow ; such case can be identified as most or least favorable arrangement of connected joints in rock mass. Such rock mass is regarded as a porous medium consisting of a set of subregions with distinct various permeabilities, whose distribution is statistically nonhomogeneous. It should be recalled that for statistical nonhomogeneous rock mass, the overall flow changes depending on the boundary conditions, and hence the prediction of a single value for effective 


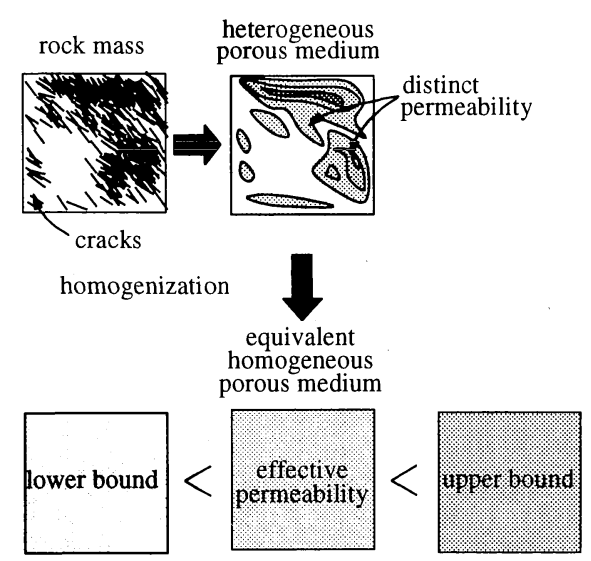

Fig.2 Modeling of rock mass as heterogeneous porous medium

permeability is meaningless.

From the view-point of the probability theory, the effective permeability is regarded as the expectation of the relation between the average flow quantities. The expectation provides an accurate evaluation when the variation of the geological data and the associated local permeabilities are relatively small. If the variation is large, however, a realization of the flow could be far from the expectation, and hence the prediction of the largest or smallest flow becomes important. When the effective permeability attains an extreme value, such flow is realized. Therefore, the range of all possible effective permeabilities must be examined even from limited information on geological data.

\section{EXACT INEQUALITIES FOR EFFECTIVE PERMEABILITY}

In this section, exact inequalities that hold for all possible realizations of flow are derived from field equations, and the range of effective permeabilities of a sample is determined. To this end, several fundamental relations are summarized for the field variables and their average quantities.

\section{( 1 ) Fundamental Relations of Rock Hyd- raulics}

Let $V$ be rock mass which consists of a set of subregions with distinct permeabilities; see Fig.2 for modeling of rock mass as a heterogeneous porous medium. This $V$ is either the whole rock mass of interest or a part of it. Each subregion, denoted by $\Omega^{\alpha}$, has permeability $\boldsymbol{k}^{\alpha}$, though its shape or location is arbitrary. The range of $\boldsymbol{k}^{\alpha \prime} \mathrm{s}$ can be huge, and they can be strongly anisotropic.

A permeability tensor field, $\boldsymbol{k}=\boldsymbol{k}(\boldsymbol{x})$, which takes $\boldsymbol{k}^{\alpha}$ in $\Omega^{\alpha}$, is then defined. As the most simple setting, it is assumed that 1) the viscosity of the fluid is 1 ;2) the flow is in stationary state ; and 3) the gravity effect is omitted. The associated field variables are pressure and velocity, $p=p(\boldsymbol{x})$ and $\boldsymbol{v}$ $=\boldsymbol{v}(\boldsymbol{x})$, which satisfy

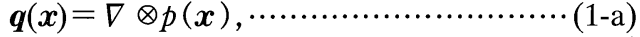

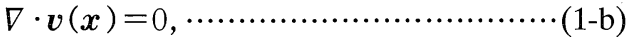

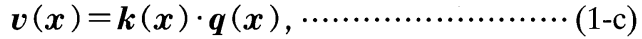

where $\nabla$ is the del operator $\left((\nabla)_{i}=\partial / \partial x_{i}\right)$, and $\otimes$ and "." denote tensor product and contraction. Namely, Eqs. $(1-\mathrm{a} \sim \mathrm{c})$ are the definition of pressure gradient, $\boldsymbol{q}=\boldsymbol{q}(\boldsymbol{x})$, the continuity equation, and the local Darcy law. It should be noted that the form of Eqs. $(1-\mathrm{a} \sim \mathrm{c})$ is arranged to correspond to that of the strain-displacement relation, the equilibrium equation, and the constitutive relation in continuum mechanics, when $p$, $\boldsymbol{q}$, and $\boldsymbol{v}$ are read as displacement, strain, and stress, respectively.

The effective permeability of $V$ is defined through the relation between the average pressure gradient and velocity. With the aid of Eqs. $(1-a, b)$ and the Gauss theory, the volume integral of $\boldsymbol{q}$ and $\boldsymbol{v}$ over $V$ can be reduced to the surface integral of pressure and velocity flux on boundary $\partial V$, i.e.,

$$
\begin{aligned}
& <\boldsymbol{q}>=\frac{1}{V} \int_{\partial V} \nu p d S, \\
& <\boldsymbol{v}>=\frac{1}{V} \int_{\partial V} \boldsymbol{x} s d S,
\end{aligned}
$$

where $s=s(\boldsymbol{x})$ is the velocity flux given by $\nu(\boldsymbol{x}) \cdot \boldsymbol{v}$ $(\boldsymbol{x})$ with $\nu(\boldsymbol{x})$ being the outer unit normal on $\partial V$; and $\langle(\ldots)\rangle$ denotes the volume average of field (...) $(x)$ taken over $V$.

The product of the velocity and pressure gradient, $\boldsymbol{v} \cdot \boldsymbol{q}$, is called flow energy rate in this paper. In addition to Eps.(2-a, b), one can deduce the following relations for the average flow energy rate:

$$
\begin{aligned}
& \langle\boldsymbol{v} \cdot \boldsymbol{q}\rangle=\frac{1}{V} \int_{\partial V} s p d S, \\
& \langle\boldsymbol{v} \cdot \boldsymbol{q}\rangle-\langle\boldsymbol{v}\rangle \cdot\langle\boldsymbol{q}\rangle \\
& =\frac{1}{V} \int_{\partial V}(s-\boldsymbol{\nu} \cdot\langle\boldsymbol{v}>)(p-\boldsymbol{x} \cdot\langle\boldsymbol{q}>) d S \text {. }
\end{aligned}
$$

Equation (3-b) corresponds to Hill's condition $^{\text {footnote2) }}$ for strain energy in continuum mechanics.

\section{(2) Exact Inequalities}

Although it is usually taken for granted that the effective permeability of $V$ is unique, the relation between $\langle\boldsymbol{q}\rangle$ and $\langle\boldsymbol{v}\rangle$ varies depending on the surface conditions on $\partial V$. Indeed, there are two limiting values of $\langle\boldsymbol{q} \cdot \boldsymbol{v}\rangle$ among all flows with common $\langle\boldsymbol{q}\rangle$ or $\langle\boldsymbol{v}\rangle$. The one limiting value is given by the flow when $V$ is subjected to the

\footnotetext{
footnote2) Eqs.(3-a, b) hold for any arbitrary pressure gradient field and divergence-free velocity field. 
uniform velocity flux boundary conditions, i.e., $s(\boldsymbol{x})=\nu(\boldsymbol{x}) \cdot \boldsymbol{V}$ on $\partial V$ with constant $\boldsymbol{V}$. The proof is straightforward. Designating the resulting fields by putting superscript $s$, the positive-definiteness of $\boldsymbol{k}$ yields

$$
0 \leq<\left(\boldsymbol{q}-\boldsymbol{q}^{s}\right) \cdot \boldsymbol{k} \cdot\left(\boldsymbol{q}-\boldsymbol{q}^{s}\right)>\text {. }
$$

Applying Eq.(3-b) to the divergence-free velocity, $\boldsymbol{v}^{s}=\boldsymbol{k} \cdot \boldsymbol{q}^{s}$, and the pressure gradient, $\boldsymbol{q}^{s}-\boldsymbol{q}=\nabla \otimes$ $\left(p^{s}-p\right)$, and taking advantage of the boundary conditions assigned for $\boldsymbol{v}^{s}$, one can compute the right side of Eq.(4) as

$$
\begin{aligned}
< & \left(\boldsymbol{q}-\boldsymbol{q}^{s}\right) \cdot \boldsymbol{k} \cdot\left(\boldsymbol{q}-\boldsymbol{q}^{s}\right)> \\
= & <\boldsymbol{q} \cdot \boldsymbol{k} \cdot \boldsymbol{q}\rangle-\left\langle\boldsymbol{q}^{s} \cdot \boldsymbol{k} \cdot \boldsymbol{q}^{s}\right\rangle \\
& \left.+2<\boldsymbol{v}^{s}\right\rangle \cdot\left\langle\boldsymbol{q}^{s}-\boldsymbol{q}>. . .\right.
\end{aligned}
$$

Hence, if the volume average of $\boldsymbol{q}$ coincides with that of $\boldsymbol{q}^{s}$, Eqs. $(4,5)$ yields

$$
\left.0 \geq<\boldsymbol{q}^{s} \cdot \boldsymbol{k} \cdot \boldsymbol{q}^{s}\right\rangle-\langle\boldsymbol{q} \cdot \boldsymbol{k} \cdot \boldsymbol{q}\rangle \text {. }
$$

According to the imposed boundary conditions, $\left\langle\boldsymbol{v}^{s} \cdot \boldsymbol{q}^{s}\right\rangle=\left\langle\boldsymbol{v}^{s}\right\rangle \cdot\left\langle\boldsymbol{q}^{s}\right\rangle$ holds, and hence $\overline{\boldsymbol{k}}$ defined as $\left\langle\boldsymbol{v}^{s}\right\rangle=\overline{\boldsymbol{k}} \cdot\left\langle\boldsymbol{q}^{s}\right\rangle$ gives $\left\langle\boldsymbol{q}^{s} \cdot \boldsymbol{k} \cdot \boldsymbol{q}^{s}\right\rangle=$ $\left\langle\boldsymbol{q}^{s}\right\rangle \cdot \overline{\boldsymbol{k}} \cdot\left\langle\boldsymbol{q}^{s}\right\rangle$. It follows from Eq.(6) that for all possible realization of flow through $V$, the above defined $\overline{\boldsymbol{k}}$ satisfies

$$
\left.0 \geq \boldsymbol{q}^{o} \cdot \overline{\boldsymbol{k}} \cdot \boldsymbol{q}^{o}-<\boldsymbol{q} \cdot \boldsymbol{k} \cdot \boldsymbol{q}\right\rangle,
$$

with $\boldsymbol{q}^{o}=\langle\boldsymbol{q}\rangle$. This inequality can be physically interpreted as follows:

among all possible flows with common average pressure gradient, the flow subjected to the uniform flux boundary conditions minimizes the average flow energy rate.

The other limiting value for the average flow energy rate can be obtained by considering the linear pressure boundary conditions, i.e., $p(x)=x$ - $\boldsymbol{Q}$ on $\partial V$ with constant $\boldsymbol{Q}$. If the resulting fields are designated by putting superscript $p$, and if $\overline{\boldsymbol{k}}$ is defined as $\left\langle\boldsymbol{q}^{p}\right\rangle=\overline{\boldsymbol{k}}^{-1} \cdot\left\langle\boldsymbol{v}^{p}\right\rangle$, then, the following inequality is derived in essentially the same manner as Eq.(7) is derived:

$$
0 \geq \boldsymbol{v}^{o} \cdot \overline{\boldsymbol{k}}^{-1} \cdot \boldsymbol{v}^{o}-\left\langle\boldsymbol{v} \cdot \boldsymbol{k}^{-1} \cdot \boldsymbol{v}>\right.
$$

with $\boldsymbol{v}^{o}=\langle\boldsymbol{v}\rangle$. Since $\boldsymbol{v}$ in Eq.(8) is an arbitrary divergence-free velocity field, the interpretation of Eq.(8) is

among all possible flows with common average velocity; the flow subjected to the linear pressure boundary conditions minimizes of the average energy flux rate.

An attention must be paid on the difference of $\overline{\boldsymbol{k}}$ used in Eqs. $(7,8)$; the former for the flow under the uniform velocity flux boundary conditions, and the latter for the flow under the linear pressure boundary conditions.

\section{BOUNDS FOR EFFECTIVE PER- MEABILITY}

Sharper bounds for the effective permeabilities of $V$ are obtained from Eqs. $(7,8)$, by using $\boldsymbol{q}$ or $\boldsymbol{v}$ which is closer to the actual field. Since direct analysis of heterogeneous $V$ is tedious, this paper applies an equivalent inclusion method to rock hydraulics to determine such field variables.

\section{(1) Equivalent Inclusion Method for Rock Hydraulics}

The equivalent inclusion method is to solve an original problem for a heterogeneous body by using the solution of an auxiliary problem for a homogeneous body where an eigenfield is prescribed $^{1,2)}$. The auxiliary problem can be solved efficiently by using the Green function for the homogeneous body. For rock hydraulics, the auxiliary problem is set for a porous medium with reference permeability $\boldsymbol{k}^{o}$ and an eigenvelocity field, $\boldsymbol{v}^{*}=\boldsymbol{v}^{*}(\boldsymbol{x})$, which replace Eq.(1-c) as

$$
\boldsymbol{v}(\boldsymbol{x})=\boldsymbol{k}^{o} \cdot \boldsymbol{q}(x)+\boldsymbol{v}^{*}(x) \text {. }
$$

The eigenvelocity corresponds to the local flow that is caused by the heterogeneity. If the permeability is smaller/larger than $\boldsymbol{k}^{o}$, positive/negative $\boldsymbol{v}^{*}$ should be induced.

Provided that $V$ is a (square or oblique) parallelepiped, ${ }^{\text {footnote } 3)}$ a disturbance pressure gradient field due to $\boldsymbol{v}^{*}(\boldsymbol{x})$ can be expressed in terms of integral operator $\boldsymbol{\Gamma}^{o}=\boldsymbol{\Gamma}^{o}\left(\boldsymbol{x} ; \boldsymbol{v}^{*}\right)$ which satisfies

$$
\begin{aligned}
& <\boldsymbol{\Gamma}^{\circ}(\boldsymbol{v})>=\boldsymbol{O}, \cdots \ldots \ldots \ldots \ldots \ldots \ldots \ldots(10-\mathrm{a}) \\
& <\boldsymbol{\Gamma}^{\circ}(\boldsymbol{v}) \cdot\left(\boldsymbol{k}^{o} \cdot \boldsymbol{\Gamma}^{o}\left(\boldsymbol{v}^{*}\right)+\boldsymbol{v}^{*}\right)>=0 . \cdots \cdots(10-\mathrm{b})
\end{aligned}
$$

This operator corresponds to the disturbance of fields at $\boldsymbol{x}$ that is caused by the existence of the heterogeneities in whole $V$. While $\Gamma^{o}$ plays an essential key role in the following subsections, its derivations are purely mathematical and omitted here; see Appendix A for the summary of derivation.

In order to relate the auxiliary problem to the original problem, one may choose reference permeability $\boldsymbol{k}^{o}$ such that $\boldsymbol{k}-\boldsymbol{k}^{o}$ be negative-semidefinite in $V$ and two pressure gradient fields, $\boldsymbol{q}(\boldsymbol{x})=\boldsymbol{q}^{o}+\boldsymbol{\Gamma}^{o}\left(\boldsymbol{x} ; \boldsymbol{v}^{*}\right)$ and $\boldsymbol{q}^{\prime}(\boldsymbol{x})=\left(\boldsymbol{k}(\boldsymbol{x})-\boldsymbol{k}^{o}\right)^{-1}$. $\boldsymbol{v}^{*}(\boldsymbol{x})$, satisfy

$$
0 \leq<\left(\boldsymbol{q}-\boldsymbol{q}^{\prime}\right) \cdot\left(\boldsymbol{k}-\boldsymbol{k}^{0}\right) \cdot\left(\boldsymbol{q}-\boldsymbol{q}^{\prime}\right)>\text {. }
$$

Equality in Eq.(11) holds if and only if $\boldsymbol{q}$ and $\boldsymbol{q}^{\prime}$ are identical, i.e.,

$$
\left(\boldsymbol{k}(\boldsymbol{x})-\boldsymbol{k}^{o}\right)^{-1} \cdot \boldsymbol{v}^{*}(\boldsymbol{x})-\boldsymbol{\Gamma}^{o}\left(\boldsymbol{x} ; \boldsymbol{v}^{*}\right)-\boldsymbol{q}^{o}=\boldsymbol{O} .
$$

Since Eq.(12) implies that the velocity field due to $\boldsymbol{v}^{*}$ is related to the pressure gradient through $\boldsymbol{k}$, it

footnote3) When $V$ is an ellipsoid of arbitrary shape, the same results can be derived ${ }^{1), 2}$. 
is equivalent to the consistency condition for the field in the auxiliary problem to coincide with those in the original problem. Therefore, as the fields produced by $\boldsymbol{v}^{*}$ approach the actual fields in $V$, the value of the right side of Eq.(11) becomes smaller.

Making use of Eqs.(10-a, b), one can rewrite the right side of Eq.(11) as $\langle\boldsymbol{q} \cdot \boldsymbol{k} \cdot \boldsymbol{q}\rangle-\boldsymbol{q}^{o} \cdot \boldsymbol{k}^{o} \cdot \boldsymbol{q}^{o}+J$, where $J=J\left(\boldsymbol{v}^{*} ; \boldsymbol{q}^{\circ}\right)$ is a functional defined as

$$
\begin{aligned}
& J\left(\boldsymbol{v}^{*} ; \boldsymbol{q}^{o}\right)=<\boldsymbol{v}^{*} . \\
& \quad\left(\left(\boldsymbol{k}-\boldsymbol{k}^{o}\right)^{-1} \cdot \boldsymbol{v}^{*}-\boldsymbol{\Gamma}^{o}\left(\boldsymbol{v}^{*}\right)-2 \boldsymbol{q}^{o}\right)>.
\end{aligned}
$$

This function gives the error of the flow energy rate which is estimated by using a trial eigenvelocity. If the trial one is closer to $v^{*}$ that satisfies Eq.(12), $\langle\boldsymbol{q} \cdot \boldsymbol{k} \cdot \boldsymbol{q}\rangle-\boldsymbol{q}^{o} \cdot \boldsymbol{k}^{o} \cdot \boldsymbol{q}^{o}+J$ approaches 0 .

It follows from Eqs. $(7,11,13)$ that the effective permeability tensor satisfies

$$
0 \geq \boldsymbol{q}^{o} \cdot \overline{\boldsymbol{k}} \cdot \boldsymbol{q}^{o}-\boldsymbol{q}^{o} \cdot \boldsymbol{k}^{o} \cdot \boldsymbol{q}^{o}+J\left(\boldsymbol{v}^{*} ; \boldsymbol{q}^{o}\right)
$$

for any arbitrary $\boldsymbol{v}^{*}$, provided that $\boldsymbol{k}-\boldsymbol{k}^{o}$ is negative-semi-definite. It is shown in Appendix $B$ that $J$ attains the global minimum for the eigenvelocity that satisfies Eq.(12).

\section{( 2 ) Computation of Bounds}

The accurate computation of $J$ requires precise information on $\boldsymbol{k}$. The property of $\boldsymbol{\Gamma}^{o}$, however, enables one to evaluate a value of $J$ for a particular class of $v^{* \prime}$ s even though the information is limited. In view of $\Omega^{\alpha \prime}$ s permeability being given by $\boldsymbol{k}^{\alpha}$, the eigenvelocity is set to take on a constant value, $\boldsymbol{v}^{* \alpha}$, in each $\Omega^{\alpha}$, i.e.,

$$
\begin{aligned}
& \boldsymbol{k}(\boldsymbol{x})=\sum_{a} \varphi^{\alpha}(\boldsymbol{x}) \boldsymbol{k}^{\alpha}, \cdots \\
& \boldsymbol{v}^{*}(\boldsymbol{x})=\sum_{\alpha} \varphi^{\alpha}(\boldsymbol{x}) \boldsymbol{v}^{* \alpha},
\end{aligned}
$$

where $\varphi^{\alpha}(x)$ is the characteristic function of $\Omega^{\alpha}$ $\left(=1\right.$ in $\Omega^{\alpha}$ and $=0$ otherwise). For these $\boldsymbol{k}$ and $\boldsymbol{v}^{*}$, functional $J$ becomes

$$
\begin{aligned}
& J\left(\boldsymbol{v}^{*} ; \boldsymbol{q}^{o}\right)=\sum_{\alpha} f^{\alpha} \boldsymbol{v}^{* \alpha} \cdot\left(\boldsymbol{k}^{\alpha}-\boldsymbol{k}^{o}\right)^{-1} \cdot \boldsymbol{v}^{* \alpha} \\
& \quad+\sum_{a, \beta}<\left(\varphi^{\alpha} \boldsymbol{v}^{* \alpha}\right) \cdot \boldsymbol{\Gamma}^{o}\left(\varphi^{\beta} \boldsymbol{v}^{* \beta}\right)>-2 \overline{\boldsymbol{v}}^{*} \cdot \boldsymbol{q}^{o} .
\end{aligned}
$$

where $f^{\alpha}$ is the volume fraction of $\Omega^{\alpha}$ in $V$, and $\overline{\boldsymbol{v}}^{*}$ is the ensemble average of $\boldsymbol{v}^{* \alpha \prime}$ s.

As shown in Appendix $C$, if reference permeability is isotropic, $\boldsymbol{k}^{o}=k^{o} \boldsymbol{I}$ with $(\boldsymbol{I})_{i j}=\delta_{i j}$, integral operator $\boldsymbol{\Gamma}^{o}$ satisfies

$$
\begin{aligned}
& <\left(\varphi^{\alpha} \boldsymbol{e}_{i}\right) \cdot \boldsymbol{\Gamma}^{o}\left(\varphi^{\beta} e_{i}\right)> \\
& =\left\{\begin{array}{ll}
-\frac{1}{k^{0}} f^{\alpha}\left(1-f^{\alpha}\right) & \text { for } \alpha=\beta \\
\frac{1}{k^{0}} f^{\alpha} f^{\beta} & \text { for } \alpha \neq \beta
\end{array},\right.
\end{aligned}
$$

where summation convention is used for suffix $i$. It should be noted that $\left(\varphi^{\alpha} \boldsymbol{e}_{i}\right) \cdot \boldsymbol{\Gamma}^{o}\left(\varphi^{\beta} \boldsymbol{e}_{i}\right)$ corresponds to the sum of the product between unit eigenveloci- ties in the $x_{i}$-direction in $\Omega^{\alpha}$ and pressure gradients produced by another unit eigenvelocity in the same direction in $\Omega^{\beta}$. Since an eigenvelocity is a source of disturbance, the left side of Eq.(17) gives the average interaction between the disturbances in $\Omega^{\alpha}$ and $\Omega^{\beta}$. Hence, Eq.(17) means that

if measured in the form of the left side of Eq.(17), the interaction between two arbitrary regions can be determined only by the volume fraction of the regions, without depending on other geometrical parameters, such as the distance, the relative orientation, or the shape.

With the aid of Eq.(17), functional $J$ given by Eq.(16) can be explicitly computed; choosing $k^{o-}$ such that $\boldsymbol{k}^{o-}=k^{o-} \boldsymbol{I}$ makes $\boldsymbol{k}^{\alpha}-\boldsymbol{k}^{o-}$ negative-semidefinite for all $\boldsymbol{k}^{\alpha \prime}$ s, and taking sum of Eq.(14) for $\boldsymbol{q}^{o}=\boldsymbol{q}^{o} \boldsymbol{e}_{i}$ and $\boldsymbol{v}^{* \alpha}=v^{* \alpha} \boldsymbol{e}_{i}(i=1,2,3)$ with common $q^{o}$ and $v^{* \alpha \prime}$ s, one can obtain

$$
\begin{aligned}
0 & \geq\left(\overline{\boldsymbol{k}}-\boldsymbol{k}^{o-}\right)_{i i}\left(q^{o}\right)^{2}+ \\
& +\sum_{\alpha} f^{\alpha}\left(\left(\boldsymbol{k}^{\alpha}-\boldsymbol{k}^{o-}\right)_{i i}^{-1}+\frac{1}{k^{o-}}\right)\left(v^{* \alpha}\right)^{2} \\
& -\frac{1}{k^{o-}}\left(\bar{v}^{*}\right)^{2}-6 \bar{v} q^{o}, \ldots \ldots \ldots \ldots \ldots \ldots \ldots \ldots \ldots \ldots \ldots
\end{aligned}
$$

where $\bar{v}^{*}$ is the ensemble average of $v^{* \alpha \prime}$ s.

The right side of Eq.(18) is a computable quadratic form (with linear terms) for $v^{* \alpha \prime}$ s. Its extreme value provides the following bound for $\overline{\boldsymbol{k}}$ :

$$
0 \geq \frac{1}{3}(\overline{\boldsymbol{k}})_{i i}-\bar{k}^{c}\left(k^{o-}\right)
$$

where $\bar{k}^{\mathrm{c}}=\bar{k}^{\mathrm{c}}$ is a function of reference permeability $k^{o}$, defined as

$$
\bar{k}^{c}\left(k^{o}\right)=\left\{\sum_{\alpha} \frac{f^{\alpha}}{k^{o}+1 /\left(\boldsymbol{k}^{\alpha}-k^{o} \boldsymbol{I}\right)_{i i}^{-1}}\right\}^{-1} .
$$

Geophysical data required to compute this $\bar{k}^{c}$ is only the volume fraction of subregions of common local permeability, i.e., $\left\{f^{\alpha}, \boldsymbol{k}^{\alpha}\right\}$. The data on location, shape, or orientation of each phase are not required.

In essentially the same manner as shown above, Eq.(8) implies the lower bound of $\bar{k}$; when $k^{\text {o+ }}$ makes $\boldsymbol{k}^{\alpha}-k^{o+} \boldsymbol{I}$ positive-semi-definite, $\bar{k}^{c}\left(k^{o+}\right)$ becomes a lower bound for $(\overline{\boldsymbol{k}})_{i i} / 3$, i.e.,

$$
0 \leq \frac{1}{3}(\overline{\boldsymbol{k}})_{i i}-\bar{k}^{c}\left(k^{o+}\right) ;
$$

see Nemat-Nasser and $\mathrm{Hori}^{1)}$ for more detailed derivation for the mechanical case.

\section{(3) Statistical Description of Local Per- meability}

The effective permeability, $\overline{\boldsymbol{k}}$, is a symmetric second-order tensor, and hence has three principle values, $\bar{k} \geq \bar{k}_{2} \geq \bar{k}_{3}$. The first invariant of this tensor is given by $(\bar{k})_{i i}=\bar{k}_{1}+\bar{k}_{2}+\bar{k}_{3}$. Therefore, Eq.(19) provides an upper bound for the maximum 
principle value, $\bar{k}_{1}$, while Eq.(21) an lower bound for the minimum principle value, $\bar{k}_{3}$. In particular, if $\overline{\boldsymbol{k}}$ is isotropic, the three principle values are the same, and the upper and lower bounds for the effective permeability are $\bar{k}^{c}\left(k^{o-}\right)$ and $\bar{k}^{c}\left(k^{o+}\right)$.

For the computation of $\bar{k}^{c}$, it suffices to get the information on a set of volume fractions and local permeabilities, $\left\{f^{\alpha}, \boldsymbol{k}^{\alpha}\right\}$. Although it is difficult to measure local permeabilities in the whole rock mass, there are often cases where distribution, orientation, or connectivity of discontinuities are evaluated in a statistical manner and the statistical distribution of local permeabilities can be given. If a probability density function for the local permeability, $f=f(\boldsymbol{k})$, is given, the bound given by Eq. (20) can be computed; function $f$ determines the probability of finding a local permeability $\boldsymbol{k}$ as $f$ $(\boldsymbol{k}) d k_{1} d k_{2} d k_{3}=f(\boldsymbol{k}) d \boldsymbol{k}$.

When $f(\boldsymbol{k})$ is given, the volume fraction of subregions with permeability $\boldsymbol{k}$ is $f(\boldsymbol{k})$. Hence, at the limit of infinitely many $\Omega^{\alpha \prime}$ s, eigenvelocities $v^{* \alpha}$ for $\Omega^{\alpha}$ in Eq.(18) is replaced by a function $v^{*}$ $(\boldsymbol{k})$ for local permeabilities $\boldsymbol{k}$, and the quadratic form of $v^{* \alpha \prime}$ s becomes a functional for $v^{*}(\boldsymbol{k})$, i.e.,

$$
\begin{gathered}
\int_{K} f(\boldsymbol{k})\left(\left(\boldsymbol{k}-\boldsymbol{k}^{o-}\right)_{i i}^{-1}+\frac{1}{k^{o-}}\right)\left(v^{*}(\boldsymbol{k})\right)^{2} d \boldsymbol{k} \\
-\frac{1}{k^{o-}}\left(\bar{v}^{*}\right)^{2}-6 \bar{v}^{*} q^{o}, \ldots \ldots \ldots \ldots \ldots \ldots \ldots \ldots \ldots
\end{gathered}
$$

where $K$ is the range of $\boldsymbol{k}$, and $\bar{v}^{*}$ is the expectation of $v^{*}(\boldsymbol{k})$. The stationary value of $J^{p}$ is given by

$$
\bar{k}^{c p}\left(k^{o}\right)=\left\{\int_{K} \frac{f(\boldsymbol{k})}{k^{o}+1 /\left(\boldsymbol{k}-k^{o} \boldsymbol{I}\right)_{i i}^{-1}} d \boldsymbol{k}\right\}^{-1} \cdots
$$

This stationary value becomes the global maximum or minimum if $\boldsymbol{k}-k^{o} \boldsymbol{I}$ is negative-semi-definite or positive-semi-definite, respectively. For given $f$ $(\boldsymbol{k})$, therefore, the bounds of the effective permeability are

$$
\bar{k}^{c p}\left(k^{o+}\right) \leq \frac{1}{3}(\overline{\boldsymbol{k}})_{i i} \leq \bar{k}^{c p}\left(k^{o-}\right)
$$

where $k^{0 \pm}$ is chosen such that $\boldsymbol{k}-k^{o \pm} \boldsymbol{I}$ be positive or negative-semi-definite for all $\boldsymbol{k}$ in $K$. It should be noted that the bounds given by Eq.(24) are the extreme value that $V$ with the given statistical distribution of local permeabilities admits. Hence, the realization that $V$ takes such extreme value is quite low, but $\boldsymbol{k}$ cannot take larger or smaller value.

\section{SIMULATION FOR PERMEABLE FLOW THROUGH HETEROGE- NEOUS MEDIA}

Numerical simulations of flow through porous media consisting of randomly generated heterogeneities and subjected to randomly varying

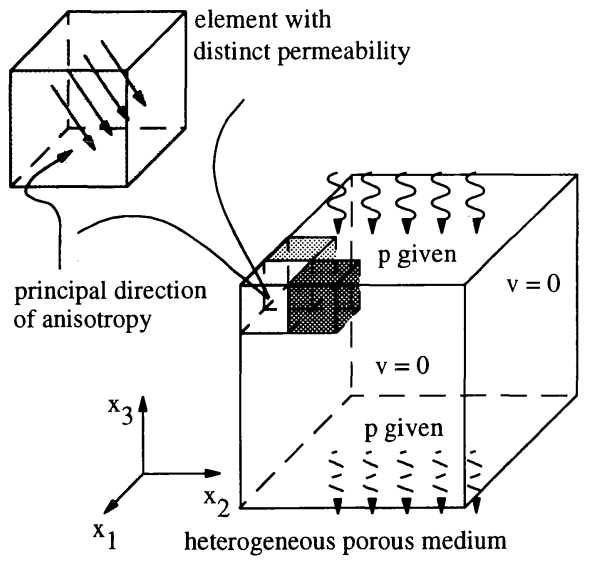

Fig.3 Schematic view of FEM analysis of heterogeneous porous medium

boundary conditions are performed, in order to compute the range for effective permeabilities. As shown in Fig.3, the following problem is set for each medium: the medium is a cube of length $L$ which consists of $I^{3}$ cubes of the common shape; each cube $E^{\alpha}$ has its distinct permeability tensor, $\boldsymbol{k}^{\alpha}$, which is characterized by the three principle values, $k_{1}^{\alpha} \geq k_{2}^{\alpha}=k_{3}^{\alpha}$, and the direction of anisotropy, which is given by the orientation of the first principle direction, is $\left(1, \phi^{\alpha}, \theta^{\alpha}\right)$ in the polar coordinates; and the boundary conditions are zero velocity fluxes on the lateral four faces, zero pressure on the bottom face, and varying pressures prescribed on the top face.

The anisotropy of $\boldsymbol{k}^{\alpha}$ is induced when majority of joints or cracks in $E^{\alpha}$ lie in particular directions, and the direction of anisotropy becomes parallel to the faces of these joints or cracks. For simplicity, however, the cases where all subregions are isotropic are considered; the principle values are set as $k_{1}^{\alpha}=k_{2}^{\alpha}=k_{3}^{\alpha}$, and $k_{1}^{\alpha} / k^{r}$ is chosen from 100 , 10 , or 1 with $k^{r}$ being a reference value, 1 . The elements with high permeability represents regions where cracks are densely and randomly distributed. In numerical simulation, these elements are randomly chosen for each $V$ as well as the pressures on the top face. The volume fraction of $E^{\alpha \prime}$ s of $k_{1}^{\alpha} / k^{\circ}=100,10$, or 1 is denoted by $f_{1}, f_{2}$, or $f_{3}=1-f_{1}$ $-f_{2}$. The estimated bounds are computed by setting $k^{0-} / k^{r}=100$ and $k^{o-} / k^{r}=1$; the bounds are plotted for $f_{1}$ and $f_{2}$ in Fig.4.

First, the effects of the boundary conditions on the effective permeability are examined. For $f_{1}=f_{2}$ $=0.25(I=4), 100 V^{\prime} \mathrm{s}$ are generated by choosing highly permeable elments randomly, and the effective permeability of each $V$ are computed for 100 different pressure boundary conditions on the top and bottom faces. The effective permeability 


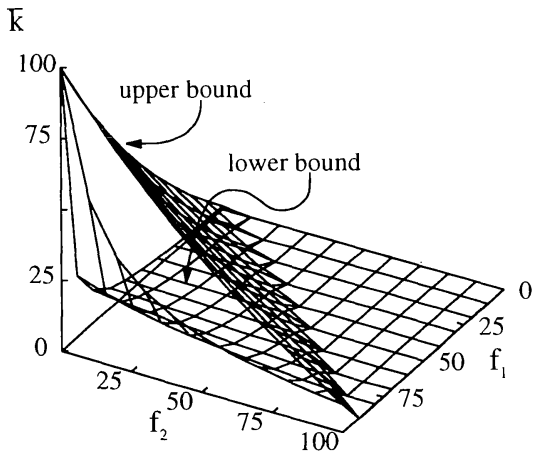

Fig.4 Estimated bounds for porous medium with isotropic elements

$\bar{k}_{33}$ is computed from the average velocity in the $x_{3^{-}}$ direction, by solving boundary-value problems with the FEM. The two typical results are shown in Fig.5. Most of $V^{\prime}$ s show relatively large range of effective permeabilities varies, and the distribution appears close to the normal distribution, like the one in Fig.5 a). For some $V$, however, the maximum value is exceptionally larger than other values as shown in Fig.5 b). This strong dependence of the effective permeability on the boundary conditions is due to the statistical heterogeneity of $V$.

Since the range of the effective permeabilities depend on both the structure of $V$ and the prescribed boundary conditions, 10000 simulations are made for a give pair of $\left(f_{1}, f_{2}\right)$, by choosing both the distribution of highly permeable elments and the distribution of pressures randomly. In Fig.6, the distribution of the computed effective permeabilities is shown for the case of $f_{1}=f_{2}=0.25$ of $I$ $=4,6,8$. The vertical axis indicates the frequency of $V^{\prime}$ s whose $\bar{k}$ falls in the indicated value. While the distribution becomes sharper as $I$ increases the minimum and maximum values remain almost the same, and they are close to the predicted bounds though they do not exceed them. As $I$ increases, it is more likely that the distribution of highly permeable elements becomes uniform. Hence, one may consider the peak value of $\bar{k}$ corresponds to the effective permeability of statistically homogeneous $V$.

As $f_{1}$ or $f_{2}$ increases, the range of $\bar{k}$ becomes wider. The range, however, still lie between the estimated bounds; four cases of $\left(f_{1}, f_{2}\right)$, the distribution of $\bar{k}$ are shown in Fig.7. It should be emphasized here that while the statistical homogeneity is not assumed nor the boundary conditions are not uniform in the simulation, the analytically obtained bounds provide reasonably accurate ranges for the computed effective per-

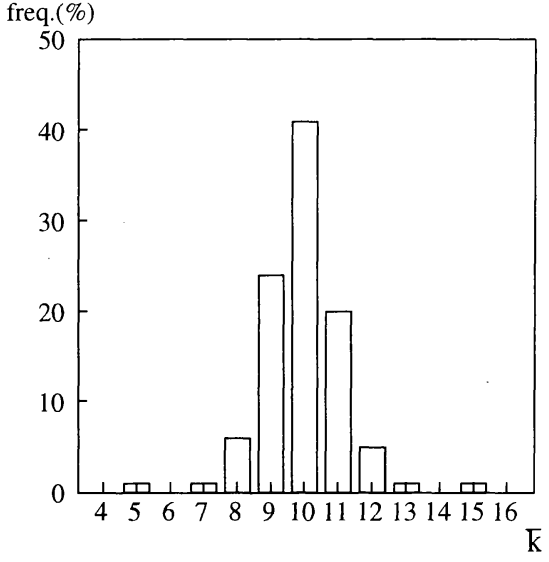

a) normal case

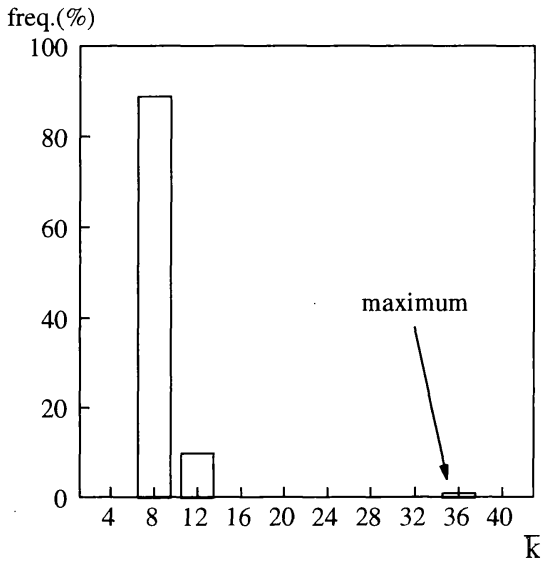

b) peculiar case

Fig.5 Effect of boundary conditions on distribution of $\bar{k}$

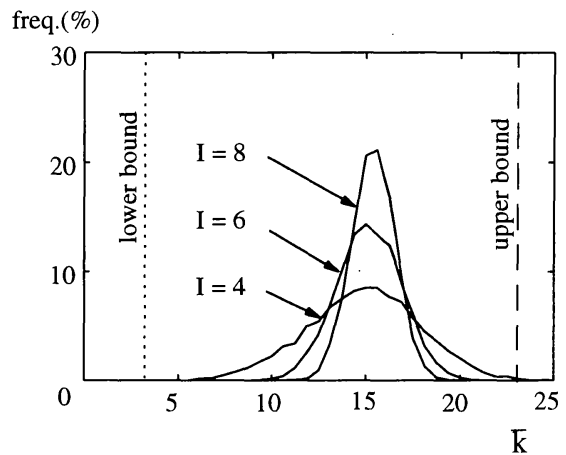

Fig.6 Distribution of effective permeabilities of media with isotropic elements with different $I$

meabilities.

In numerical simulation, it is observed that the pattern of the flow when the effective permeability is near the bounds differs from that when the effective permeability is in the middle of the range. For the case where the effective permeability is 


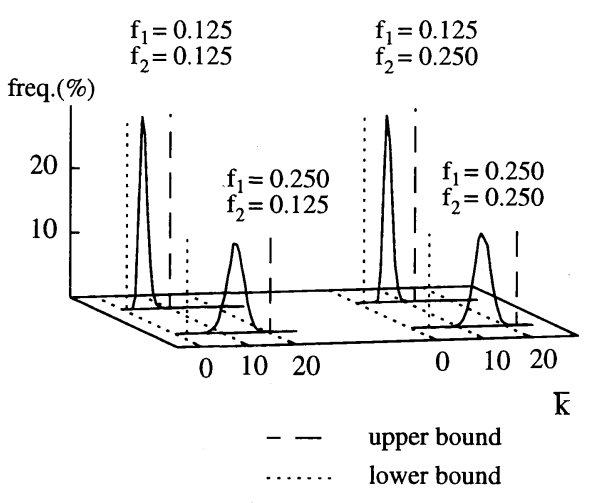

Fig.7 Distribution of effective permeabilities of media with isotropic elements of different volume fractions

close to the upper bound, a strong flow takes place in a narrow region, i.e., it appears that the flow runs through the channel made of highly permeable elements. For the case where the effective permeability is close to the lower bound, the flow does not run through highly permeable elements even though the channel of the highly permeable element is constructed. As a matter of course, the distribution of such elements are not uniform in these cases.

To visualize the pattern, the simulation is reduced to the two-dimensional setting with anisotropic elements. Square $V$ consisting of $I^{2}$ square elements $E^{\alpha \prime}$ s is used, and each element with second-order symmetric permeability tensor $\boldsymbol{k}^{\alpha}$ with the principle values $k_{1}^{\alpha} \geq k_{2}^{\alpha}$; see Hori et. $a l .{ }^{19)}$ for a more detailed explanation. Two typical distributions of flow velocities in the extreme case that yields the effective permeability close to the upper bound or the lower bound are shown in Fig.8; the length and orientation of an arrow on each element indicate the amount and direction of the average permeable flow running through the element. It is clearly shown in Fig. 8 a) that large flow runs from the top right to the bottom left, which passes channels made of highly permeable elements. In Fig. 8 b), however, such localized flow is not constructed.

\section{CONCLUSIONS}

Bounds for the effective material properties are obtained for a material which is statistically nonhomogeneous. While the permeability of a rock mass is considered, the method of computing the bounds can be applied to other properties such as elasticity or thermal conductivity. If these bounds are used in analysis of a structure made of the material, the best or worst case of the structure performance can be predicted. It should be
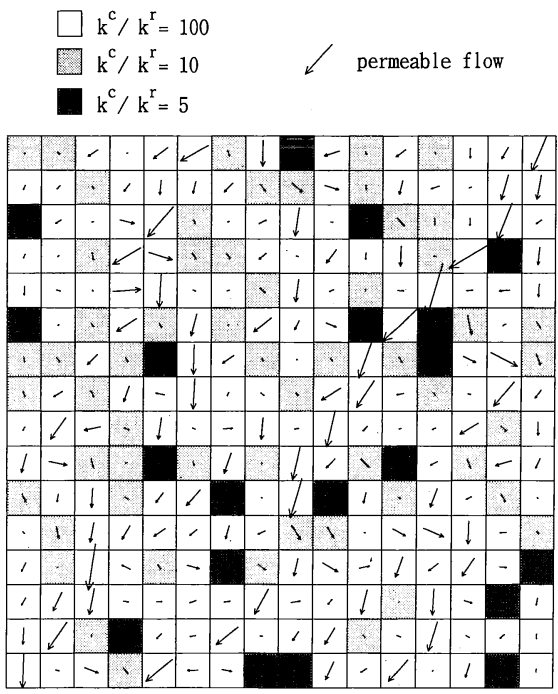

a) case of high effective permeability
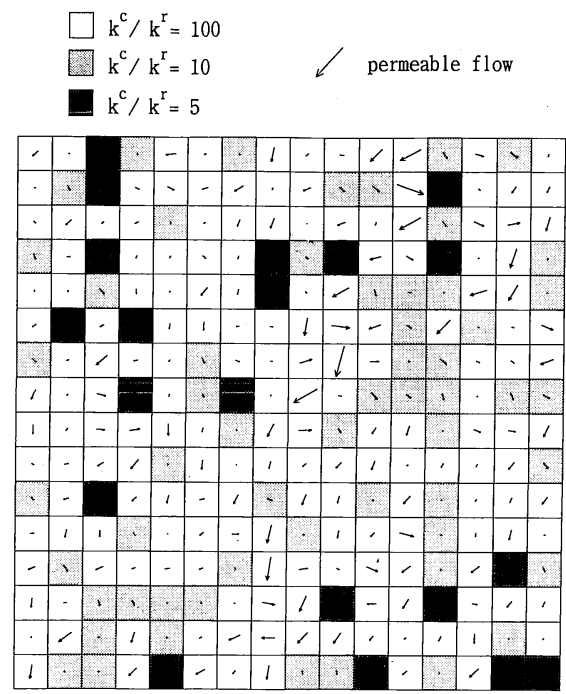

b) case of low effective permeability

Fig.8 Distribution of flow velocities in elements of heterogeneous medium

emphasized here that the range of the bounds depends on the quality and quantity of the informations on the heterogeneities, and the range becomes sharper as better informations are available. Therefore, in order to estimate the material property with the desired accuracy, one may judge the precision of the measurement that is required to make the predicted bounds lie within a range which is determined from the accuracy. 


\section{APPENDIX A APPLICATION OF EQUIVALENT INCLUSION METHOD TO ROCK HYDRAULICS}

Let $V^{*}$ be a homogeneous porous medium, with reference permeability $\boldsymbol{k}^{o}$ and eigenvelocities $\boldsymbol{v}^{*}$, subjected to the periodic boundary conditions. From Eqs.(1-a, b, 9), the governing equation for $p$ in $V^{*}$ for prescribed $\boldsymbol{v}^{*}$ is

$$
\nabla \cdot\left(\boldsymbol{k}^{o} \cdot(\nabla \otimes p(\boldsymbol{x}))+\nabla \cdot \boldsymbol{v}^{*}(\boldsymbol{x})=0 \cdots\right.
$$

Expansion of $p$ and $\boldsymbol{v}^{*}$ in the Fourier series yields the solution of Eq.(A.1), and the pressure gradient field produced by $\boldsymbol{v}^{*}$ is given by

$$
\begin{aligned}
& \boldsymbol{\Gamma}^{o}\left(\boldsymbol{x} ; \boldsymbol{v}^{*}\right)=\sum_{\xi \neq 0} \mathscr{F} \boldsymbol{\Gamma}^{o}(\xi) \cdot \mathscr{F} \boldsymbol{v}^{*}(\xi) \exp (\xi \cdot \boldsymbol{x}), \\
& \mathscr{F} \boldsymbol{\Gamma}^{\circ}(\xi)=\frac{\xi \otimes \xi}{\xi \cdot \boldsymbol{k}^{o} \cdot \xi}
\end{aligned}
$$

where $\xi$ is determined from the edges of cubic $V^{*}$ and $\mathscr{F} \boldsymbol{v}^{*}(\boldsymbol{\xi})=\left\langle\boldsymbol{v}^{*}(\boldsymbol{x}) \exp (-\boldsymbol{\xi} \cdot \boldsymbol{x})\right\rangle$ is the Fourier series coefficients. A constant pressure gradient field is added to $\boldsymbol{\Gamma}^{o}$, since it is a homogeneous solution of Eq.(A.1).

Equations (10-a, b) are easily derived from explicit form of $\boldsymbol{\Gamma}^{o}$. First, kernel $\exp (\boldsymbol{\xi} \cdot \boldsymbol{x})$ for $\boldsymbol{\xi} \neq$ $\boldsymbol{O}$ satisfies

$$
<\exp (\xi \cdot x)>=0 \text {, }
$$

and hence the volume average of each term in the sum of $\boldsymbol{\Gamma}^{o}$ vanishes. This leads to Eq.(10-a). Next, by definition, $\mathscr{F} \boldsymbol{\Gamma}^{o}$ satisfies

$$
\begin{aligned}
& \mathscr{F} \boldsymbol{\Gamma}^{o}(-\xi)=\mathscr{F} \boldsymbol{\Gamma}^{o}(\xi) \ldots \ldots \ldots \ldots \ldots \\
& \mathscr{F} \boldsymbol{\Gamma}^{o}(\xi) \cdot \boldsymbol{k}^{o} \cdot \mathscr{F} \boldsymbol{\Gamma}^{o}(\xi)=\mathscr{F} \boldsymbol{\Gamma}^{o}(\xi)
\end{aligned}
$$

Thus, $\mathscr{F} \boldsymbol{\Gamma}^{o}(-\xi) \cdot\left(\boldsymbol{k}^{o} \cdot \mathscr{F} \boldsymbol{\Gamma}^{o}(\xi)-\boldsymbol{I}\right)$ vanishes, and hence the volume average of $\boldsymbol{v} \cdot \boldsymbol{q}$ always becomes zero. This leads to Eq.(10-b).

\section{APPENDIX B FUNCTIONAL FOR EIGENVELOCITY}

Functional $J$ given by Eq.(13) is of the essentially the same form as the functional used in the HashinShtrikman variational principle, which is based on the assumption of the statistical homogeneity ${ }^{9), 11)}$ and requires some approximation to compute its value. However, $J$ is derived without making any special assumptions and is computable.

Since $\boldsymbol{\Gamma}^{o}$ is explicitly given, the property of $J$ can be discussed without introducing additional physical assumptions or approximations. First, it is seen that operator $\left\langle\boldsymbol{v}^{*} \cdot \boldsymbol{\Gamma}^{o}(\boldsymbol{v})\right\rangle$ is self-adjoint for its argument $v^{*}$, and hence the first variation of $J$ becomes

$$
\begin{aligned}
& \delta J\left(\boldsymbol{v}^{*} ; \boldsymbol{q}^{o}\right)=<\delta \boldsymbol{v}^{*} \\
& \quad \cdot\left(\left(\boldsymbol{k}-\boldsymbol{k}^{o}\right)^{-1} \cdot \boldsymbol{v}^{*}-\boldsymbol{I}^{o}\left(\boldsymbol{v}^{*}\right)-\boldsymbol{q}^{o}\right)>\ldots . .
\end{aligned}
$$

The Euler equation of $J$ coincides with Eq.(12), which can be rewritten as

$$
\begin{aligned}
\left.\boldsymbol{k}^{o} \cdot\left(\boldsymbol{q}^{o}\right)+\boldsymbol{\Gamma}^{o}\left(\boldsymbol{x} ; \boldsymbol{v}^{*}\right)\right)+\boldsymbol{v}^{*}(\boldsymbol{x}) \\
\quad=\boldsymbol{k}(\boldsymbol{x}) \cdot\left(\boldsymbol{q}^{o}+\boldsymbol{\Gamma}^{o}\left(\boldsymbol{x} ; \boldsymbol{v}^{*}\right)\right) . \cdots
\end{aligned}
$$

This is the consistency condition.

The consistency condition has a unique solution, for which $J$ becomes (locally) stationary. When $\boldsymbol{k}-$ $\boldsymbol{k}^{o}$ is negative-semi-definite, the stationary value becomes the global maximum. This is easily seen from how $J$ is constructed in Section 3. Or it can be shown from the negative-semi-definiteness of $<\boldsymbol{v}^{*}$ $\cdot \boldsymbol{\Gamma}^{o}\left(\boldsymbol{v}^{*}\right)>$; with the aid of Eqs. $(10-\mathrm{a}, \mathrm{b}),\left\langle\boldsymbol{v}^{*} \cdot \boldsymbol{\Gamma}^{o}\right.$ $\left(\boldsymbol{v}^{*}\right)>$ is written as

$$
<\left(\boldsymbol{v}-\boldsymbol{k}^{o} \cdot \boldsymbol{q}\right) \cdot \boldsymbol{\Gamma}^{o}>=-<\boldsymbol{\Gamma}^{o} \cdot \boldsymbol{k}^{o} \cdot \boldsymbol{\Gamma}^{o}>,
$$

and is negative-semi-definite. Hence, functional $J$ is concave, and the stationary value is the global maximum. In a similar manner, it is shown that the functional has the global minimum when $\boldsymbol{k}-\boldsymbol{k}^{o}$ is positive-semi-definite.

\section{APPENDIX C PROPERTIES OF IN- TEGRAL OPERATOR}

When $\boldsymbol{k}^{o}$ is isotropic, $\boldsymbol{k}^{o}=k^{o} \boldsymbol{I}$, Eq.(A.3-b) becomes $\mathscr{F} \Gamma^{o}(\xi)=\xi \otimes \xi /\left(k^{o} \xi \cdot \xi\right)$, and it satisfies

$$
\boldsymbol{e}_{i} \cdot \mathscr{F} \boldsymbol{\Gamma}^{o}(\xi) \cdot \boldsymbol{e}_{i}=\mathscr{F} \boldsymbol{\Gamma}_{i i}^{o}(\xi)=\frac{1}{k^{o}} \text {. }
$$

Then, it follows from Eq.(A.3-b) that $\boldsymbol{e}_{i} \cdot \boldsymbol{\Gamma}^{o}\left(\varphi^{\beta}\right.$ $\left.\boldsymbol{e}_{i}\right)$ is

$$
\begin{aligned}
\boldsymbol{e}_{i} \cdot \boldsymbol{\Gamma}^{o}\left(\boldsymbol{x} ; \varphi^{\beta} \boldsymbol{e}_{i}\right)= & \sum_{\boldsymbol{\xi} \neq O}\left(\boldsymbol{e}_{i} \cdot \boldsymbol{\Gamma}^{o}(\xi) \cdot \boldsymbol{e}_{i}\right) \\
& <\varphi^{\beta}(\boldsymbol{y}) \exp (\xi \cdot(\boldsymbol{x}-\boldsymbol{y}))> \\
= & \frac{1}{k^{o}}\left(\varphi^{\beta}(\boldsymbol{x})-f^{\beta}\right) ; \cdots \cdots(\mathrm{C} .2)
\end{aligned}
$$

this is becauase $\left\langle\varphi^{\beta}(\boldsymbol{y}) \exp (-\boldsymbol{\xi} \cdot \boldsymbol{y})\right\rangle$ is the Fourier series coefficient of the characteristic function, and $\left\langle\varphi^{\beta}\right\rangle$ becomes $f^{\beta}$. Therefore, the volume average of Eq.(C.2) yields

$$
\begin{aligned}
& <\left(\varphi^{\alpha} \boldsymbol{e}_{i}\right) \cdot \boldsymbol{\Gamma}^{o}\left(\varphi^{\alpha} \boldsymbol{e}_{i}\right)> \\
& =\frac{1}{k^{o}}<\varphi^{\alpha}\left(\varphi^{\beta}-f^{\beta}\right)>v .
\end{aligned}
$$

This relation leads to Eq.(17).

\section{REFERENCES}

1) Nemat-Nasser, S. and Hori, M. : Micromechanics : Overall Properties of Heterogeneous Solids, Northholland, New York, 1983.

2) Hori, M. and Nemat-Nasser, S. : Universal bounds for overall elastic moduli of heterogeneous materials, Trans. Roy. Soc. London (to be published).

3) Bear, J. and Buchlin, J.M. : Modeling and Applications of Transport Phenomena in Porous Media, Kluwer, Dordrecht, 1987. 
4) Bear, J., Tsang, C-F., and Marsily, G. : Flow and Contaminant Transport in Fractured Rock, Academic Press, San Diego, 1993.

5) Oda. M. : Permeability tensor for discontinuous rock masses, Geotechnique, Vol.35, No.4, pp.483 495, 1985.

6) Oda, M. : An equivalent continuum model for coupled stress and fluid flow analysis in jointed rock masses, Water Resources Research, Vol.22, No.13, pp.1845 1856, 1986.

7) Yamabe, T., Hara, N. and Oda, M. : Parameter determination in elastic analysis of jointed rock masses by crack tensor analys, Proc. of JSCE, Vol.282/III-7, pp.121 129, 1987.

8) Long, J.C.S. and Witherspoon, P.A. : The relationship of the degree of interconnection to permeability in fracture networks, J. Geophysical Res., Vol.90, No.B 4, pp.3087 3098, 1985.

9) Hashin, Z. and Shtrikman, S. : A variational approach to the theory of the elastic behavior of polycrystals, J. Mech. Phys. Solids, Vol.10, pp.343 352, 1962.

10) Hashin, Z. and Shtrikman, S. : A variational approach to the theory of the magnetic permeability of multiphase materials, J. Appl. Phys., Vol.33, pp.3125 3131, 1962.

11) Willis, J.R. : Bounds and self-consistent estimates for the overall properties of anisotropic composites, J. Mech. Phys. Solids, Vol.25, pp.185 202, 1977.

12) Torquato, S. : Random heterogeneous media : microstruc- ture and improved bounds on effective properties, Appl. Mech. Rev., Vol.42, No.2, pp.37 76, 1991.

13) Willis, J.R. : Variational and related methods for the overall properties of anisotropic composites, Appl. Mech. Rev., Vol.21, pp.1 78, 1981.

14) Hashin, Z. : Analysis of composite materials-A survey, $J$. Appl. Mech., Vol.50, pp.481 505, 1983.

15) Beran, M.J. : Statistical Continuum Theories, Interscience, New York, 1968.

16) Franfort, G.A. and F. Murat, F. : Homogenization and optimal bounds in linear elasticity, Archive Rat. Mech. and Analysis, Vol.94, pp.307 334, 1986.

17) Milton, G.W. : On characterizing the set of possible effective tensors of composites : the variational method and the translation method, Communications on Pure and Applied Mathematics, Vol.43, pp.63 125, 1990.

18) Milton, G.W. and Kohn, R. : Variational bounds on the effective moduli of anisotropic composites, J. Mech. Phys. Solids, Vol.36, pp.597 629, 1988.

19) Hori, M., Yamaguchi, E. and Hosokawa, M. : Study on effective permeability of cracked rocks, J. Struct. Eng. (in Japanese, accepted).

20) Mura, T. : Micromechanics of Defects in Solids, Nijhoff, New Yori, 1987.

(Received January 25, 1994)

\section{統計的非均一材料の有効物性の限界値について}

\section{堀 宗朗}

不均一材料の有効物性の推定は重要な課題であるが，材料構造が一様でない場合や不 均一性に関する情報が少ない場合, 種々の条件によって有効物性が変わるため, 推定が 困難となる．この問題に対処するため，本研究は，与えられた情報から，構造が一様で ない材料の有効物性が取り得る範囲を推定する手法を提案する. 岩盤浸透問題を例に, 有効透水係数の範囲を厳密に推定し, 妥当性の検討のため, 数值シミュレーションを行っ た. シミュレーションで得られた有効透水係数の分布は推定された範内囲に納まってお り，提案された有効物性の範囲推定手法の妥当性が示唆される. 